Universite de Lausanne, France, Thèse de Doctorat., 1997

${ }^{6}$ Elsohly H N, Croom E M, Elesohly M A. Analysis of the antimalarial sesquiterpene artemisinin in Artemisia annua by high-performance liquid chromatography with postcolumn derivatization and ultraviolet detection. Pharmaceutical Research. 1987; 4: 258-60

\section{Preparation and activity of diterpenoids against trypomastigotes of Trypanosoma cruzi}

Jacqueline A. Takahashi ${ }^{{ }^{*}}$; Henriete S. Vieira ${ }^{1}$; Eliane A. Silva ${ }^{1}$;
Maria A. D. Boaventura ${ }^{\text {; }}$ Alaíde B. de Oliveira ${ }^{2}$; Egler Chiari ${ }^{3}$

${ }^{1}$ Departamento de Química, ICEx, Universidade Federal de Minas Gerais, Av. Antônio Carlos, 6627, 31270-901, Belo Horizonte, MG, Brazil

2Departamento de Produtos Farmacêuticos, Faculdade de Farmácia, UFMG, Av. Olegário Maciel, 2360, 30180-112, Belo Horizonte, MG

${ }^{3}$ Departamento de Parasitologia, ICB, UFMG, Av. Antônio Carlos, 6627, CP 2486, 31270-901, Belo Horizonte, MG jacque@Ag.arizona.edu

\begin{abstract}
A systematic investigation on the trypanocidal effect of several natural products isolated from Brazilian plant species has been carried out. In this paper we report on the results obtained from the screening of 26 diterpenes from natural sources or of synthetic/microbial transformations origin (mainly derivatives of kaurenoic acid) against trypomastigote forms of Trypanosoma cruzi, the causative agent of Chagas'disease. In the in vitro assays, kaurenoic acid, kaurenol, acutifloric acid and stemodin showed a complete elimination of parasites from the blood. Therefore, such diterpenoids can be considered as starting materials for molecular modification in the search for lead compounds for clearance of infected blood to be used in transfusions. Blood previously treated with active compounds was submitted to an infectivity test. Samples proceeded from treatment with kaurenol and kaurenoic acid showed to be completly clean from $T$. cruzi as no infection was observed in mice inoculated with contaminated blood treated by these compounds.
\end{abstract}

During the last decades, many efforts have been done aiming at the discovery of a suitable substitute of gentian violet for sterilization of chagasic blood. Among the active compounds resulting from these works, amphiphilic cationic drugs showed in vitro activity against trypomastigotes of Trypanosoma cruzi $^{1}$. However, none of these compounds was able to substitute gentian violet and new lead compounds are still to be found.

Kaurenoic acid (1), a diterpenoid that presents some interesting biological activities ${ }^{2}$, has been reported as active against $T$. cruzi $^{3}$. However, we have observed that, besides its low solubility in the media used for the biological assay, its activity is accompanied by some erythrocytes lysis. The trypanocidal activity of kaurenoic acid (1) was firstly described with no reference to the erythrocyte lysis ${ }^{3}$, that has been systematically observed in our experiments. In the same work, sodium and triethylammonium salts of (1) were prepared and 
tested, but no improvement of the trypanosomicidal activity was observed.

We have synthetised some hydrochlorides salts derivatives of kaurenoic acid (1). One of them was able to reduce erythrocyte lysis, but without improvement of the trypanocidal activity compared to kaurenoic acid.

In this work we report the biological screening of several derivatives (and related compounds) of kaurenoic acid (1), obtained from plant sources or by chemical/microbial transformation, in the search for compounds active against $T$. cruzi without the inconvenience caused by erythrocytes damage.

Complete elimination of $T$. cruzi trypomastigotes was observed for 4 , out of the 26 tested compounds: kaurenoic acid (1), kaurenol (3), acutifloric acid (21) and stemodin (25). The $\mathrm{ED}_{100}$ for these compounds are shown in Table 1. Lysis of erythrocytes was again observed for the experiment with kaurenoic acid. When compared to the gentian violet activity, all tested compounds showed lower activity. However, in the "in vivo" infectivity tests, used to check absence of trypomastigotes in samples persistently negative in "in vitro" assays (see Material and Methods), two compounds showed to be promising, kaurenol (3) and kaurenoic acid (1) as a complete clearance of blood trypomastigotes was confirmed. Mice infection was observed for those inoculated with blood treated with acutifloric acid (21) and stemodin (25).

Table 1. In vitro activity (ED $100 ; \mathrm{mg} / \mathrm{ml})$ of diterpenoids against trypomastigote forms of Trypanosoma cruzi Y strain

\begin{tabular}{l|l}
\hline Compound & $\mathrm{ED}_{100}(\mu \mathrm{g} / \mathrm{ml})$ \\
\hline Gentian Violet & 130 \\
\hline Kaurenoic acid (1) & 1.363 \\
\hline Kaurenol (3) & 1.386 \\
\hline Acutifloric acid (21) & 1.599 \\
\hline Stemodin (25) & 1.390 \\
\hline
\end{tabular}

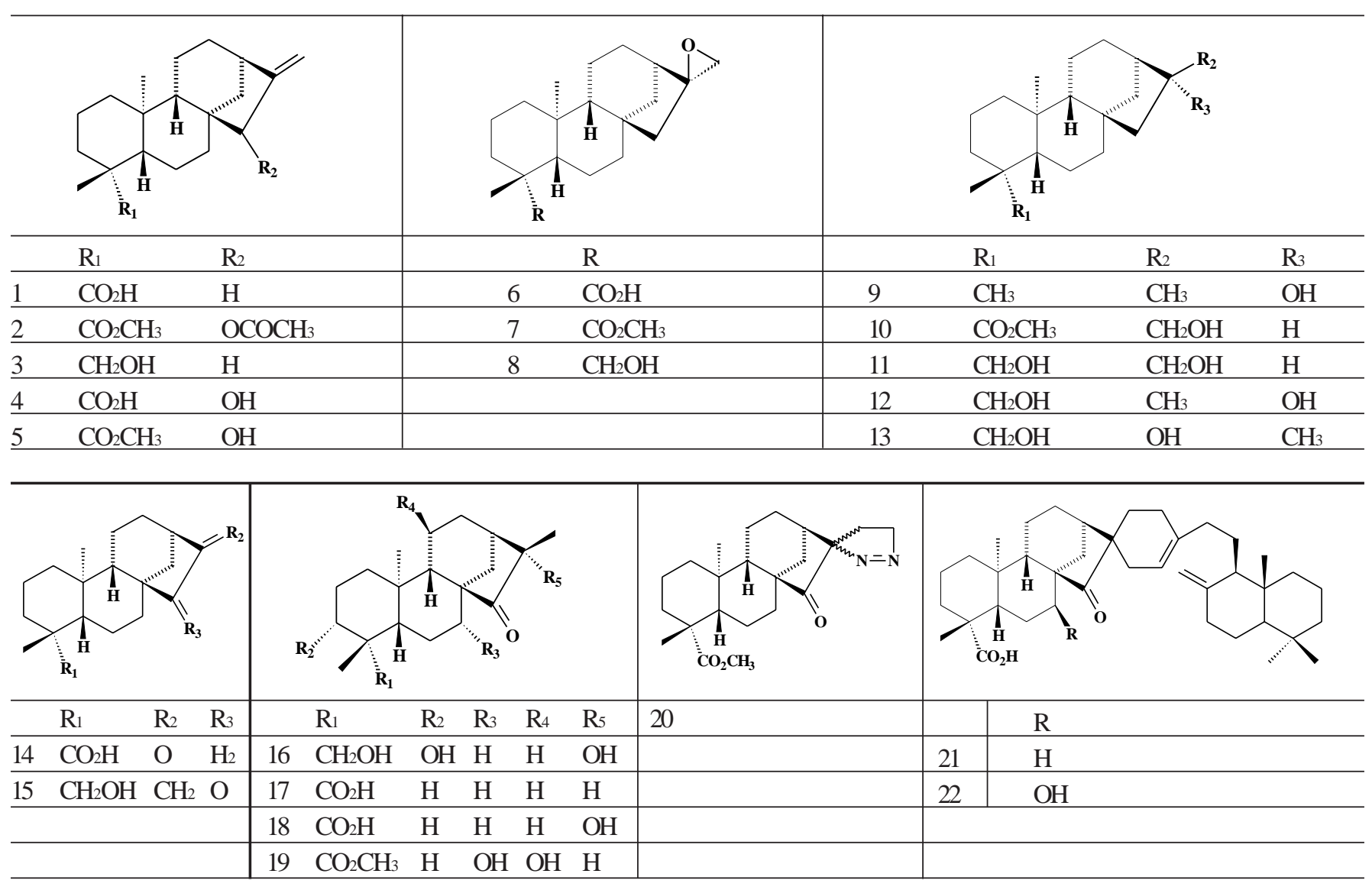

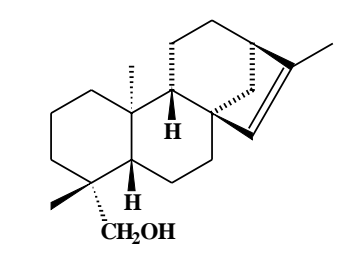

23

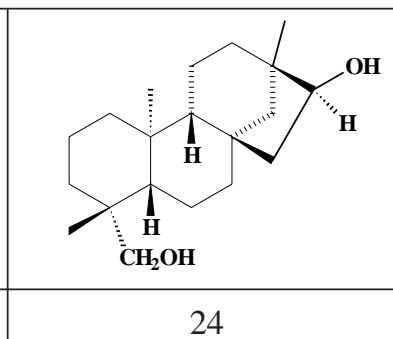

24
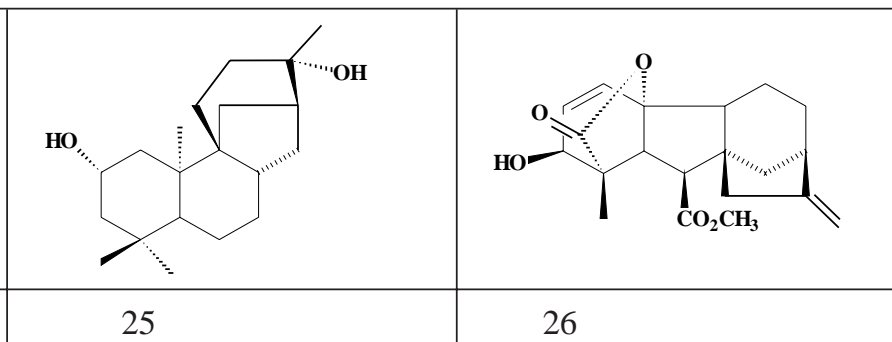
Partial activity, indicated by a reduction in the parasites number in relation to untreated control, was observed for isosteviol (24), kauranol (9), 15-hydroxykaurenoic acid (4) and its methyl ester (5), methyl ester of xylopic acid (2) and 16,17epoxy-19-kauranol (8). The remaining compounds showed no trypanocidal effect. Active and partially active diterpenes are of interest for novel chemical and/or microbial transformations in the search for more effective trypanocidal compounds. Such structural modifications of these compounds are interesting since some of them have antifungic activity ${ }^{4}$ and it is know that some antifungal drugs are potent antiproliferative agents against $T$. cruzi $i^{5}$.

\section{Material and Methods}

Of the 26 tested diterpenoids, 6 were obtained by our research group on Natural Products at the Departamento de Química and Faculdade de Farmácia, UFMG, Belo Horizonte,

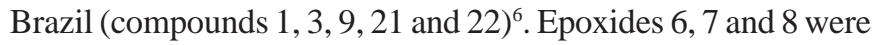
prepared by reaction with MCPBA in $\mathrm{CHCl}_{3}$ followed by purification on silica gel column chromatography. Compounds $4,12,13-20$ were prepared by chemical and/or microbial transformations. Spectroscopic data of these compounds have been published elsewhere ${ }^{7,8,9}$. Methyl esters (2, 5, 7, 10 and 19) were prepared of the corresponding acids by reaction with diazomethane . Stemodin (25) was kindly supplied by Dr. P. B. Reese and Dr. M.R. Wilson (University of West Indies, Jamaica). Compound 26 was prepared from gibberellic acid which was purchased from Aldrich Chemical Company. Dihydroxyisosteviol (24) and compound 11 were prepared from the corresponding methyl esters by reduction with $\mathrm{LiAlH}_{4}$ in dry THF. Compound 10 was prepared from kaurenoic acid by reaction with $\mathrm{NaBH}_{4} / \mathrm{BF}_{3} / \mathrm{THF}$ and compound 23 was obtained from the acid isomerization of kaurenoic acid.

In vitro biological assays were performed according to Chiari et $\mathrm{al}^{10}$. In vivo infectivity tests were used to check the absence of trypomastigotes in those samples persistently negative in tests. Samples of blood that showed absence of trypomastigotes, after treatment with the diterpenoids, were inoculated to outbred male Swiss albino mice (18-20 g of body weight). T. cruzi blood stream trypomastigotes forms were used as an infective sample. After 7 days, hemoculture (LiT medium) and serology (indirect fluorescent antibody test) were performed in those animals which did not develop parasitemia.

\section{Acknowledgments}

The authors thank CNPq and FAPEMIG (Brazil) for fellowships and financial support.

\section{References}

${ }^{1}$ Hammond DJ, Hogg L, Gutteridge WE. Trypanosoma cruzi: Possible Control of Parasite Transmission by Blood Transfusion Using Amphiphilic Cationic Drugs. Experimental
Parasitology. 1985; 60:32-42

${ }^{2}$ Ghisalberti EL. The Biological Activit of Naturally Occurring Kaurane Diterpenes. Fitoterapia 1997; LXVIII: 303-325

${ }^{3}$ Alves TMA, Chaves PPG, Santos LMST, Nagem TJ, Murta SMF, Geravolo IP, Romanha AJ, Zani CL. A Diterpene from Mikania obtusata Active on Trypanosoma cruzi. Planta Med. 1995; 61: 85-87

${ }^{4}$ Roque NF, Giannella TL, Giesbrecht AM, Barbosa RCSBC. Kaurene Diterpenes from Wedelia paludosa. Rev. Latinoam. Quim. 1987; 18: 110-111

${ }^{5}$ Urbina JA, Lazard K, Aguirre T, Piras MM, Piras R. Antiproliferative Effects and Mechanism of Action of ICI 195,739, and Novel Bis-triazole Derivative, on Epimastigotes and Amastigotes Forms of Trypanosoma (Schizotrypanum) cruzi. Antimicrob. Agents Chemother. 1991; 35: 730-735

${ }^{6}$ Takahashi JA, Boaventura MAD, Bayma JC, Oliveira AB. Frutoic Acid, a Dimeric Kaurane Diterpene from Xylopia frutescens. Phytochemistry. 1995; 40: 607-609

${ }^{7}$ Hanson JR, Hitchcock PB, Takahashi JA. Biotransformation of ent-16b,19-dihydroxykaurane by Cephalosporium aphidicola. Phytochemistry. 1995; 40: 797-800

${ }^{8}$ Boaventura MAD, Hanson J., Hitchcock PB, Takahashi JA. The Biotransformation of ent-19-hydroxykaur-16-en-15-one by Cephalosporium aphidicola. Phytochemistry. 1994; 37:387-389 ${ }^{9}$ Oliveira AB, Hanson JR, Takahashi JA. The Biotransformation of ent-15-oxokaur-16-en-19-oic Acid and its Methyl Ester by Cephalosporium aphidicola. Phytochemistry 1995; 40: 439-442 ${ }^{10}$ Oliveira AB, Saúde DA, Perry KSP, Duarte DS, Raslan DS, Boaventura MAD, Chiari E. Trypanocidal Sesquiterpenes from Lychnophora Species. Phytotheraphy Research. 1996 10: 292295 